\title{
Commemorating 75 years of microemulsion
}

\author{
Animesh Kumar Rakshit ${ }^{1}$, Bappaditya Naskar ${ }^{2}$ and Satya Priya Moulik,* \\ ${ }^{1}$ Indian Society for Surface Science and Technology, Department of Chemistry, Jadavpur University, Kolkata 700 032, India \\ ${ }^{2}$ Department of Chemistry, Sundarban Hazi Desarat College, University of Calcutta, Pathankhali 743 611, India
}

\begin{abstract}
The first scientific paper on microemulsion was published in 1943 by Schulman and Hoar of Cambridge University. Microemulsion is basically made up of oil, water, surfactant and a short-chain alcohol, acid or amide (called 'cosurfactant'). They were called swollen micelles or by some other names but not microemulsion in 1943 or after. It was rechristened 'microemulsion' in 1959 by Schulman et al. who were then at New York's Columbia University. Research in microemulsion became popular in 1980 s when there loomed a large spectre of oil scarcity and its eventual effect on human civilization as we are aware today. Microemulsion was thought to be important for tertiary oil recovery. It has also been found to be useful for nanoparticle synthesis, in pesticide formulation, in drug delivery, and in many other ways, and the research on it still has a great potential and importance. In the 75 year of its innovation (and documentation in the literature), a short but succinct article with its flavour and importance is presented here.
\end{abstract}

Keywords: Co-surfactants, ionic liquids, microemulsion, surfactants, Winsor systems.

Microemulsions are thermodynamically stable, microheterogeneous dispersions of two immiscible liquids, oil and water, between each other in presence of surfactants and co-surfactants. In this definition, any liquid which is immiscible with water is taken as 'oil'. Two non-aqueous immiscible liquids can also form non-aqueous microemulsion. The first scientific publication on microemulsion was in 1943 (ref. 1), though the term 'Microemulsion' was first introduced in 1959 (ref. 2), and in a review article published in 1955 (ref. 3). The 1943 paper was 'Letters to the Editors' and was published on 24 July of that year in Nature which was just a little more than one page. In this paper the transparent oil continuous system was termed 'soluble oil'. It was also called 'oleopathic hydro-micelle'. The affiliations of authors were Metallurgical Laboratories (T. P. Hoar) and Department of Colloid Science (J. H. Schulman; Figure 1) of Cambridge University, UK. However, in 1959, the paper in Journal of Physical Chemistry was published from Columbia University, New York, USA where Schulman relocated from Cambridge in 1957. Schulman was born in Brazil in 1904, educated in England, worked in Cambridge and

\footnotetext{
*For correspondence. (e-mail: spmcss@yahoo.com)
}

Columbia and died in a tragic drowning accident in Italy in June 1967.

Hoar and Schulman ${ }^{1}$ suggested the presence of high soap/water ratio as well as the presence of 'an alcohol, fatty acid, amine or other non-ionized amphipathic substances in mol-fraction approximately equal to that of the soap'. It should be noted that much before Hoar and Schulman's paper, people in Australia were familiar with a transparent dispersion which had eucalyptus oil, soap, water and spirit. In 1940s, flavour oil-based microemulsions such as cola, cream soda were in the market ${ }^{4-6}$. An oil/water microemulsion known as 'Dri-brite', a carnauba wax emulsion which was useful as floor polish, leather polish was in the market from 1928 onwards $^{4}$. Pine oilbased microemulsion which was used as bactericide, fungicide, etc. was also in the market ${ }^{7}$. This dispersion was used to clean wool. The interfacial tension (IFT) between oil and water is very high. That is why they are insoluble in each other. Since the free energy of formation of oilwater interface is positive, they do not spontaneously mix.

Presence of a surfactant in the system can lower IFT to a large extent. However, quite often one needs to add short-chain alcohol or acid or amine in the system to lower IFT to assist formation of microemulsion making the free energy of formation negative due to entropic factors. The following thermodynamic equation is applicable

$$
\Delta G=\Delta H-T \Delta S+\gamma \Delta A,
$$

where $G, H, T, S, \gamma$ and $A$ are free energy, enthalpy, temperature in degree $\mathrm{K}$, entropy, IFT and interfacial area

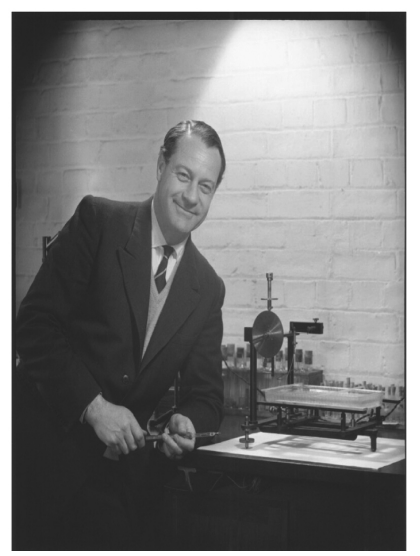

Figure 1. Jack Henry Schulman (1904-1967). 
respectively. In case of microemulsion formation, $\Delta H$ is almost zero, $\gamma$ being quite small, $\gamma \Delta A$ is not large though $\Delta A$ is expected to be large. Hence, high positive entropy change makes the magnitude of eq. (1) negative, and the system becomes thermodynamically stable. Prince ${ }^{8}$ suggested that IFT could have negative values and if so, then both $T \Delta S$ and $\gamma \Delta A$ become negative and hence $\Delta G$ becomes negative in eq. (1) leading to a spontaneous microemulsion formation. However, it has been shown that the negative surface tension is not essentially required for both micro- or macroemulsion formation ${ }^{9}$. Recently, Kaptay ${ }^{10}$ has shown that surface tension can become negative only for solutions with strong repulsion between the components. Negative surface tension is possible in a non-equilibrium state for a macroscopic solution. He suggested that for a nano-solution negative surface tension is possible even in equilibrium state. Bialke et al. ${ }^{11}$ and Patashinski et al. ${ }^{12}$ have discussed the negative surface tension for an unstable interface. The size of oil droplets in oil-in-water $(\mathrm{o} / \mathrm{w})$ or water droplets in waterin-oil (w/o) microemulsion is quite small $\sim 10-100 \mathrm{~nm}$. In case of emulsions the droplet sizes are $\sim 10^{5} \mathrm{~nm}$, and the magnitude of eq. (1) becomes positive. Emulsion is not thermodynamically stable; with time emulsion is expected to separate out in two phases and hence it is said to be kinetically stable. Agitation of the system, i.e. providing outside energy is required for non-spontaneous emulsion formation whereas microemulsion formation is a spontaneous process. Some confusion is observed in the literature where different terms like 'nano emulsion', 'mini emulsion', etc. are used. Microemulsion is generally completely transparent or slightly bluish in colour or translucent. Normally, micelles are assemblies of surfactants in aqueous medium. Microemulsions are assemblies of water, oil, surfactants and co-surfactants (not always inclusively though); they are either (surfactant + cosurfactant) stabilized dispersions of w/o or o/w. The w/o microemulsion may also be categorized as inverse micelle. Both micelle and inverse micelle (microemulsion) are shown in Figure 2. It may be interesting to note that Prince's article (ref. 4, chap. 1) is titled as 'Schulman's microemulsion'.

In inverse (or reverse) micelle, water (surfactant + cosurfactant) and a non-polar organic liquid are present. Without water inverse micelle is not formed. The
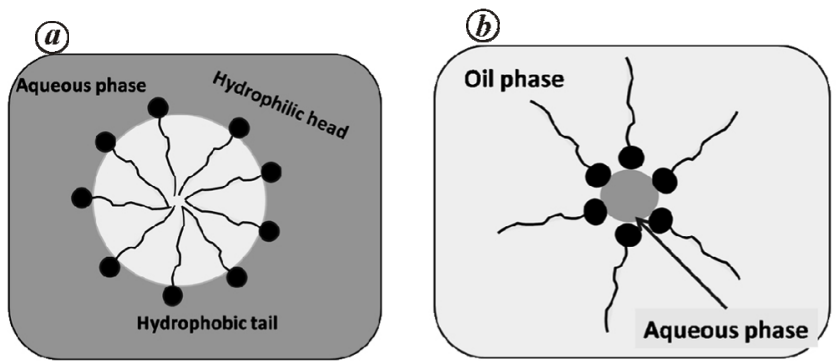

Figure 2. Schematic representations of (a) micelle and (b) inverse micelle or 'microemulsion'. ratio of water/surfactant is taken to be the criterion in distinguishing between inverse micelle and microemulsion. If this ratio is greater than 10 , the assemblies are microemulsions; for lower values they are called inverse micelles $^{13}$. Sometimes ${ }^{14}$ this value is also taken to be 15 . Generally, a co-surfactant is required for an ionic surfactant, but for surfactant like Aerosol OT (AOT) ${ }^{15-18}$, and some non-ionic surfactant like Triton X-100 (TX-100), etc. ${ }^{19,20}$, co-surfactants may not be always necessary. Amount of surfactant necessary in the formation of microemulsion, is quite high.

There are several review articles in the literature which have comprehensively presented and discussed the formation of microemulsions, their physico-chemical properties, phase diagrams, percolating phenomenon, uses in nanoparticle formation, as drug carrier, in cosmetics, in petroleum industry and in various other fields ${ }^{21-27}$. In this article, we present a comprehensive discussion on some of these properties of microemulsions, and their important uses and applications with reference to specific examples.

\section{Preparation and phase behaviour of microemulsion}

Microemulsions are formed spontaneously, and they are thermodynamically stable. Generally, water, surfactant and co-surfactant mixture at various ratios is titrated with oil until a turbidity is observed. This is taken as the end point of titration. One can also take water and oil mixture in various proportions (which is turbid) and titrate it with a surfactant-co-surfactant mixture where the end point is the disappearance of turbidity. In practice, any combination can be used and the end point is either appearance or disappearance of turbidity. The weight percentage of oil, water and surfactant-co-surfactant mixture can then be computed and plotted in a triangular pseudo-ternary phase diagram. In this phase diagram various regions can be observed as shown in Figure 3. Depending upon the concentration and nature of co-surfactant and oil, different types
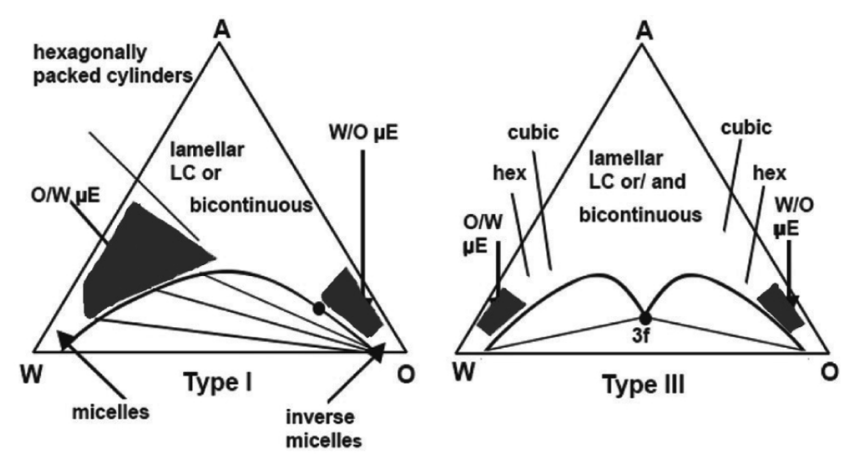

Figure 3. A representative pseudo-ternary phase diagram with various structural regions. A, amphiphile; $\mathrm{W}$, water; $\mathrm{O}$, oil; $\mu \mathrm{E}$, microemulsion. 
of structures can be observed in pseudo-ternary phase diagram. It is seen from these figures that as the concentration of oil increases, o/w microemulsion changes over to w/o microemulsion passing through a bicontinuous microemulsion system. That means there is a phase inversion which indicates that there is change in the curvature of the $\mathrm{o} / \mathrm{w}$ interface, and at the lowest curvature a bicotinuous system is obtained.

Broadly two different regions are observed for microemulsions: monophasic and biphasic. The biphasic region is emulsion where kinetic stability is important whereas the monophasic region may contain $\mathrm{w} / \mathrm{o}$ and $\mathrm{o} / \mathrm{w}$ microemulsions as well as other types of dispersions. The region map may be much more complicated than is shown here: it becomes a function of the nature of oil, surfactant, co-surfactant and also the nature of any added material (additive). The $\mathrm{o} / \mathrm{w}$ and $\mathrm{w} / \mathrm{o}$ microemulsions have different microstructures, and are not in equilibrium with each other; they may be in contact with some other species with different microstructures. Winsor divided microemulsions into four different groups depending upon their microstructures ${ }^{28,29}$. It should however be noted that when Winsor made these divisions in 1948, the term 'microemulsion' was not in vogue. He used the terms 'hydrotropy', 'emulsification', 'solubilization', etc., but not 'microemulsion'. These divisions are known as Winsor I (W I), where spherical droplets of oil are dispersed in water which is in contact with oil, o/w. In Winsor II (W II), spherical droplets of water are dispersed in oil which is in contact with water, w/o. Winsor III (W III) is the system where both $\mathrm{o} / \mathrm{w}$ and $\mathrm{w} / \mathrm{o}$ dispersions are in equilibrium with what is known as bicontinuous (BC) microemulsion. There is another system which is known as Winsor IV (W IV) where either only w/o or only o/w is present. The bicontinuous structure (W III) is an extensive intertwined structure as shown in Figure 4. Figure 4 also shows the type of microemulsion formed as surfactant hydrophilic-lipophilic balance (HLB) changes. Temperature change also changes the nature of microemulsion $^{30}$.

Formulation of microemulsion depends upon many factors. One rough rule for emulsion formation is that the phase in which the stabilizing agent is more soluble will



Figure 4. Different types of Winsor systems. Type IV is not shown because it is a monophasic system. $\boldsymbol{a}$, Oil phase; $\boldsymbol{b}$, bicontinuous (middle) phase; $c$, water phase. be the external phase ${ }^{31,32}$. Griffin's simple HLB concept was a useful tool though it had serious limitation ${ }^{33,34}$. This was a numerical rating scheme in which numbers were assigned to various surfactants and the HLB number of the surfactant suggested the type of emulsion one does get. Griffin's approach was more or less empirical in nature and was based on his experience as cosmetic chemist. Davies ${ }^{35}$ modified the above method and developed HLB group numbers which on addition provided HLB number of the surfactant. However, in this case the total HLB value is obtained by adding 7 to the above computed HLB number. For non-ionic surfactants, HLB is computed differently ${ }^{35}$. Rest remains as in the case of Griffin's approach ${ }^{36}$. Winsor suggested a $R$ ratio concept which dealt with molecular interaction energies at interface and this provided an idea of phase behaviour. Though it was qualitative in nature, it did embrace all sorts of variables in the system ${ }^{37}$ which Griffin or Davies ideas did not. The $R$ ratio was expressed as $R=\left(A_{\mathrm{co}} / A_{\mathrm{cw}}\right)$, where $A_{\text {co }}$ and $A_{\text {cw }}$ are interaction energies per unit surface area between surfactant at the interface and oil, and interaction energies per unit surface area between surfactants at the interface and water respectively. That is $A_{\text {co }}$ is the interaction energy between the hydrophobic group of the surfactant, and the hydrophobic oil. $A_{\mathrm{cw}}$ is the interaction energy between the hydrophilic group of the surfactant and water. Later it was suggested that

$$
R=\left[\left(A_{\mathrm{co}}-1 / 2 A_{\mathrm{oo}}-1 / 2 A_{\mathrm{LL}}\right) /\left(A_{\mathrm{cw}}-1 / 2 A_{\mathrm{ww}}-1 / 2 A_{\mathrm{HH}}\right)\right],
$$

where $A_{\mathrm{oo}}, A_{\mathrm{LL}}, A_{\mathrm{ww}}, A_{\mathrm{HH}}$ are interaction energies between two oil molecules, two lipophilic groups, two water molecules and two hydrophilic groups respectively ${ }^{38}$. If $R<1$, it is $\mathrm{o} / \mathrm{w}$, whereas if $R>1$, it is w/o microemulsion. If $R=1$, it will be W III type ${ }^{39}$. Surfactant affinity difference (SAD) and hydrophilic lipophilic deviation (HLD) can also be used to get a microemulsion formulation idea. SAD is defined as equal to $R T \ln K_{\text {wo }}$, where $K_{\text {wo }}$ is the partition coefficient of the surfactant between water and oil. For ionic surfactants, for optimum formulation $\mathrm{SAD}=0$. For non-ionic surfactants this is not applicable, and HLD concept is used which is

$$
\mathrm{HLD}=\left[\left(\mathrm{SAD}-\mathrm{SAD}_{\mathrm{ref}}\right) / R T\right]
$$

Division by $R T$ makes HLD a unit less quantity, and condition for optimum formulation is $H L D=0$. Between SAD and HLD, the latter is a more generalized formulation criterion which includes all sorts of variables present in the system ${ }^{39}$.

\section{Microstructure of microemulsion}

Microstructure is a nm length scale (or a little higher) structure inside a bulk material, in this case, the bulk microemulsion. Different microstructures have been 
observed, viz. w/o, o/w and bicontinuous structures described in Winsor groupings. Various methodologies have been used in their studies. They are: small angle X-ray scattering (SAXS), small angle neutron scattering (SANS), dynamic light scattering (DLS), conductance, viscosity, transmission electron microscopy (TEM), Fourier transform pulse grade spin-echo NMR (FTPGSE-NMR) technique, nuclear magnetic resonance (NMR), etc. Ultra low IFT is a must for microemulsion formation and this is measured by using a spinning drop tensiometer or by using 'sessile drop' technique. The TEM experiment can be done under two different conditions: (a) cryo-TEM where microemulsion is fast freezed and that is directly observed under cold microscope, and (b) freeze fracture TEM technique where the specimen is observed at room temperature. In FT-PGSE-NMR technique, the self-diffusion coefficients of the components are measured. From the self-diffusion coefficient the degree of binding of the co-surfactant to the micelles can be understood. By using PGSE-NMR, Giustini et al. ${ }^{40}$ proved that Schulman's titration method ${ }^{2,3}$ of preparing microemulsion quantitatively describes all titration steps that finally yield microemulsion. It is also important to note that the non-ionic surfactants are quite soluble both in water and oil. At low temperature, they are reasonably soluble in water whereas at high temperature they are comparatively well soluble in oil. Increased temperature changes the character of non-ionic surfactant from hydrophilic to a lipophilic one, and influences changes in the nature of microemulsion. At low temperature a W I system is observed, it changes over to W III and W II with increase in temperature. A plot of temperature $\mathrm{T}$ against mass fraction of the surfactant in a pseudo three component system, keeping all other mass fraction constant, reveals a map as shown in Figure 5, which is often called the 'fish diagram'41. The $\bar{X}$ is the point where $1 \Phi, 2 \Phi$ and $3 \Phi$ regions intersect, and the corresponding temperature is termed phase inversion temperature (PIT). It is also termed as 'hydrophil-lipophil balance temperature' because at this temperature hydrophilicity and lipophilicity



Figure 5. Schematic plot of temperature versus surfactant concentration (Fish diagram). of the non-ionic surfactants are same. It is also called 'tricritical point'. It also indicates that $3 \Phi$ systems only exist within a temperature region, and beyond this region $2 \Phi$ systems exist. Also at any temperature, if the surfactant concentration is over the tricritical point, the $1 \Phi$ and $2 \Phi$ systems either exist individually or they coexist.

Surfactants form a monolayer at w/o interface as well as at the interface present in microemulsion. This monolayer at the interface has local curvature which determines the nature of microemulsion. Though the droplet size in $1 \Phi$ situation is dependent on the conservation of components, in the $2 \Phi$ region, it is a function of temperature dependent spontaneous curvature ${ }^{42}$. Vollmer suggested a simple rule to semi-quantitatively compute the phase boundaries in case of a non-ionic microemulsion ${ }^{43}$. According to her, both temperature and composition dependent phase diagrams can be computed for both strong and weak surfactants. The paper deals with non-ionic surfactants but suggests that this could also be used in case of ionic surfactants where the curvatures are determined by the concentration of salt and co-surfactant. For more details see reference 43. Surfactants containing 18-carbon atom chains are highly hydrophobic. By introducing 'lipophilic linker' to the hydrocarbon chain, its effective length can be increased to balance hydrophilicity ${ }^{44}$. Some such lipophilic linkers are dodecanol, ethyl oleate, etc. ${ }^{45}$. Properties of hydrophilic and amphiphilic linkers are also discussed in the literature ${ }^{46,47}$.

\section{Structural evaluation from conductometry}

Structural features of w/o microemulsions can also be evaluated by conductometry. The radius of water pool, effective radius of the droplets, their number per unit volume, effective surface area of the water pool, and the aggregation number of the surfactant and co-surfactant molecules per droplet at the o/w interphase can be estimated $^{48}$. It is known that for hydrated dispersions, appropriate extent of water binding is required to test the validity of the effective medium theory (EMT) equation of Bruggeman ${ }^{49,50}$. The extents of hydration of polyethylene glycols (PEGs) were determined from the measurement of conductance of a $0.01 \mathrm{M} \mathrm{NaCl}$ in PEG solution, and the validity of Bruggeman equation for them was confirmed. The equation was then applied to evaluate the extents of hydration of different $\mathrm{o} / \mathrm{w}$ microemulsion systems, derived from both aliphatic and aromatic oils and stabilized by non-ionic surfactants (TX-100 and Tween 20) and co-surfactnts (1-butanol, 1-hexanol and $n$-hexylamine $)^{50}$. Based on conductance percolation results of a good number of w/o microemulsion systems, a detailed analysis of the validity of EMT, EMT with dipole-dipole interaction (EMTDD), and Bemasconi-Weismann (BW) theory has been made. It has been found that most of the systems obey either EMT or EMTDD (chain) formalism 
whereas only few follow EMTDD (cluster) and BW formalisms. The results suggest that the internal structure of microemulsions can be either isolated, randomly dispersed spheres or spheroidal aggregates formed by dipolar interaction ${ }^{51}$. Detailed structural and other physicochemical aspects of various kinds of microemulsions are described in the earlier review ${ }^{52}$.

\section{Structural and related thermodynamic analysis from dilution method}

Fundamental understanding of w/o microemulsion formation and related thermodynamics is determined from a simple experimental method, called the 'Dilution method $^{3,53-55}$. In this method, w/o microemulsion of a fixed composition is destabilized by adding a known amount of oil, and then restabilized by titrating it with a co-surfactant; these destabilization and restabilization processes are performed on the system several times. The collected data of the moles of oil, and the moles of cosurfactant added in all the sets are then processed in terms of several derived relations to evaluate the distribution constant $\left(K_{\mathrm{d}}\right)$ of co-surfactant at the water droplet interface and in the bulk oil. $K_{\mathrm{d}}$ is a measure of transfer of co-surfactant from bulk oil to the interface to make a stable microemulsion, and hence a measure of the corresponding free energy change (i.e. $-R T \ln K_{\mathrm{d}}$ ). The temperature effect on $K_{\mathrm{d}}$ then helps to find out the enthalpy and entropy changes of the said transfer process in the usual way, making all the thermodynamic parameters of the water droplets formed in the system available. In addition to thermodynamics, dilution data can also be used to find out the structural information of microemulsion, viz. number of droplets per ml, surface area of droplet, number of surfactant molecules (ions) per droplet, number of co-surfactant molecules (ions) per droplet, radius of water pool in the droplet, effective area of the droplet, etc. ${ }^{56,57}$. So it is a potential method for characterizing w/o microemulsion. However, this method is not applicable to characterizing $\mathrm{o} / \mathrm{w}$ microemulsion because of various restrictions. Details can be found in the references mentioned above and other citations presented therein.

\section{Properties of microemulsion}

Ionic conductance in $\mathrm{o} / \mathrm{w}, \mathrm{w} / \mathrm{o}$ and $\mathrm{BC}$ microemulsions can be completely different. It is almost like a normal aqueous medium in $\mathrm{o} / \mathrm{w}$ microemulsion, very low in $\mathrm{w} / \mathrm{o}$, and significantly high in a $\mathrm{BC}$ microemulsion medium. In w/o microemulsion, water content and temperature are the controlling factors for the magnitude of conductance. The conductance behaviour of $\mathrm{BC}$ microemulsion is comparable with the percolation feature. It is considered that the microemulsion system passes through a BC-like stage during the process.
The phenomenon of conductance percolation in colloidal solution, i.e. w/o microemulsion is an interesting phenomenon. Under certain conditions like temperature and concentration, the droplets containing surfactant ions come to a threshold distance wherein transfer of charge between them occurs efficiently causing significant increase in conductance of the solution called as 'dynamic percolation'. Thus, the phenomenon is augmented either by increase in temperature at a fixed droplet concentration, or by the increasing concentration of dispersion at a fixed temperature. The w/o microemulsions formed with non-ionic amphiphiles containing soluble salts in the aqueous core can exhibit conductance percolation as well. Extensive studies on conductance percolation of w/o microemusions can be found in the literature ${ }^{52}$.

For W I systems, conductance values are quite high. For W III systems also, they are reasonably high. But for W II, they are low and may exhibit percolation features described above. The increase in conductance can be of few orders of magnitude.

Fang and Venable ${ }^{58}$ have shown that the water droplet radius $\left(R_{\mathrm{W}}\right)$ in w/o microemulsion can be described by the relation

$R_{\mathrm{w}}=3\left[M_{\mathrm{s}}+r M_{\mathrm{cs}}+\left\{\left(W_{\mathrm{w}}+W_{\mathrm{cs}}\right) / W_{\mathrm{s}}\right\} M_{\mathrm{s}} /\left\{N \rho_{\mathrm{d}}\left(A_{\mathrm{s}}+r A_{\mathrm{cs}}\right)\right\}\right]$,

where $M, W, \mathrm{~s}, \mathrm{cs}, \rho_{\mathrm{d}}, A$ and $N$ are molar mass, weight fraction, surfactant, co-surfactant, dispersed phase density, cross sectional area, Avogadro number respectively; $r$ is called structural ratio, and is equal to the ratio of number of molecules of surfactant and co-surfactant $\left(n_{\mathrm{s}} / n_{\mathrm{cs}}\right)$.

Figure 6 shows dependence of conductance on temperature of a w/o percolating microemulsion system. It is considered that as the temperature increases, the dispersed water droplets come in contact with each other, forming a continuous phase, exchanging the matter and thereby increasing the conductance. Similar fate arises with increasing the microemulsion concentration at a fixed

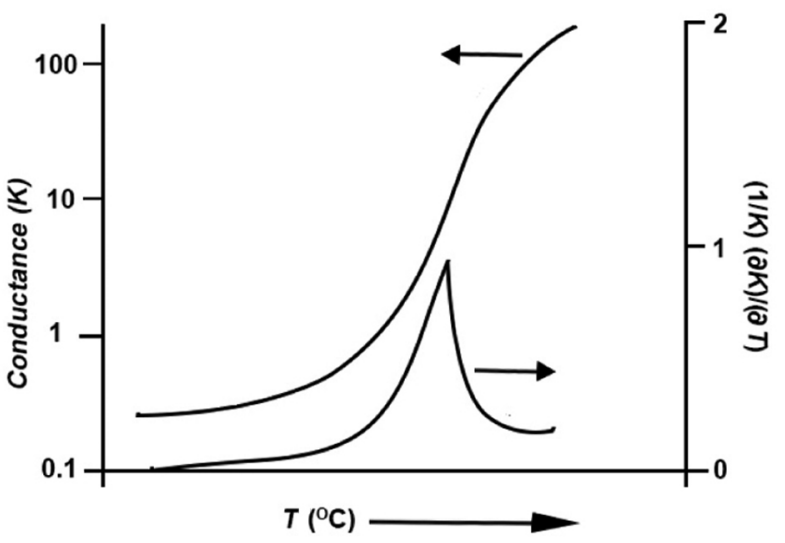

Figure 6. Schematic representation of percolation phenomenon. 
temperature. Due to ion transfer, a sigmoidal $\ln \kappa-T$, or $\ln \kappa-\omega$ (volume fraction of water) plot is obtained. The point of maximum gradient is taken as the 'threshold temperature', or 'threshold volume fraction of water' 59,60 respectively. Some researchers have proposed the use of sigmoidal Boltzmann equation to compute the threshold $T$ or $\omega$, and other corresponding quantities ${ }^{61,62}$. The sigmoidal Boltzmann equation is

$$
\begin{aligned}
\log \kappa= & \log \kappa_{\mathrm{f}}\left[1+\left\{\left(\log \kappa_{\mathrm{i}}-\log \kappa_{\mathrm{f}}\right) / \log \kappa_{\mathrm{f}}\right\}\right. \\
& \left.\times\left\{1+\exp \left(T-T_{\mathrm{c}}\right) / \Delta T\right\}^{-1}\right],
\end{aligned}
$$

where $\mathrm{i}, \mathrm{f}$ and $\mathrm{c}$ are initial, final and percolation stages respectively. It was considered that the earliest publications explaining the conductance percolation were by Lagues et $a l .^{63,64}$. It has been noted that the conductance percolation follows the scaling law

$$
\kappa=A\left(T_{\mathrm{c}}-T\right)^{-\mathrm{s}}
$$

and

$$
\kappa=B\left(T-T_{\mathrm{c}}\right)^{1},
$$

where eq. (6) holds below percolation (i.e. $T_{\mathrm{c}}>T$ ), and eq. (7) holds above percolation (i.e. $T_{\mathrm{c}}<T$ ) ranges respectively; $A$ and $B$ are free parameters; s and 1 are critical exponents. For further details on percolation, the review article of Paul et al. ${ }^{65}$ may be consulted. During percolation the dispersed stable water droplets cluster, and assist transfer of ionic charges among the droplets. The thermodynamics of droplet clustering was assessed taking droplet sizes into consideration, and the temperature effect on the process ${ }^{61,66}$. The endothermic enthalpy change of clustering was found to be moderate and the related positive entropy change to be large. The resultant state of clustering including its surroundings became disorganized or random. We refer ref. 65 for a detailed discussion on this aspect.

Viscosity provides first hand information on the internal consistency of colloidal dispersions, as well as furnish knowledge on the overall geometry of particles of the dispersed phase. Both increase and decrease in viscosity as well as a maximum are observed depending on the composition of microemulsions and environmental conditions. The ternary systems of water/surfactant/alkanol or benzyl alcohol or poly-propylene glycol, etc. can produce isotropic, low viscous solutions ranging from water to the oil corner in the ternary phase diagram, called the Lphase. This phase exhibits distinct structural, viscous and conductivity behaviours. Viscosity may also show similar type of behaviour like conductance obeying scaling laws ${ }^{31}$. The percolation of conductivity is more clearly observed for conductivity of water than for conductivity of oil. However, as viscosity of water is not sufficiently different from the viscosity of oil, parallel manifestation of percolation of viscosity like conductance is not seen. Microemulsions produced by the dispersion of polar and viscous non-aqueous solvents like glycerol and low molecular weight polyethylene glycols have the ability to show percolation of viscosity. Increase in microemulsion viscosity with temperature, essentially for AOT-derived systems, has been attributed to the phenomenon of percolation. Paul and Moulik ${ }^{67}$ and Moulik et al. ${ }^{68}$ studied a biological microemulsion consisting of water-AOThexylamine-saffola and noted that the Walden rule (constancy of conductance and viscosity product) does not hold good. Several other researchers also observed the same phenomenon ${ }^{69-72}$. The Walden rule does, however, seem to violate the Curie-Prigogine principle ${ }^{23,73}$ regarding the validity of coupling of conductance with viscosity.

It may be noted that droplet geometry (spherical or otherwise) and solvation of w/o microemulsions are understood from the measurements of intrinsic viscosity. Such results are also found in the literature ${ }^{48,68,74,75}$. Microemulsions are complex in nature, hence processing of viscosity data in the light of a unique approach is not expected. As the rheological problems of microemulsions are by no means simple, attempts have been made to treat the experimental results using different fitting procedures with the help of adjustable interaction parameters. The viscosity behaviours of w/o microemulsions at different volume fractions of water were examined using different proposed equations, viz. Eiler, Vand, Thomas and Moulik, and found to obey fairly or moderately. Such studies in the literature are less ${ }^{52}$.

\section{Types of microemulsion}

\section{Surfactant-free microemulsion}

In general, surfactant (or amphiphile) is a necessary component of microemulsions. But microemulsions may also be formed without traditional surfactants. Two immiscible fluids and an 'amphi-solvent' may give rise to nanodispersions (like microemulsions), and they are called 'surfactant-free microemulsions' (SFMEs) (Figure 7) ${ }^{76}$.

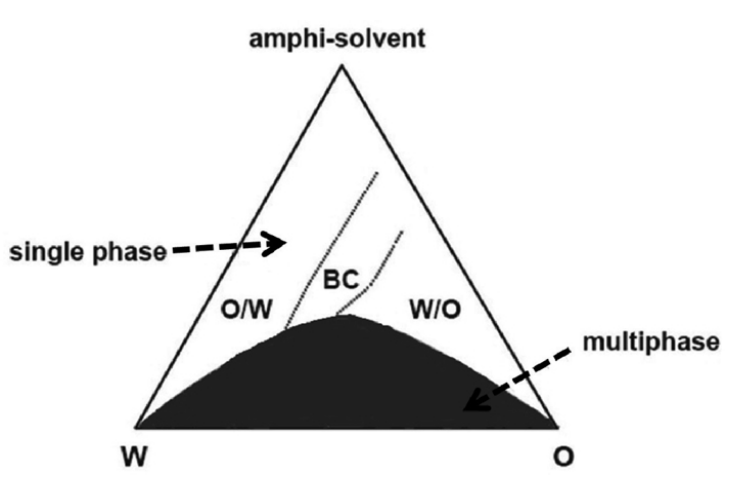

Figure 7. A schematic phase diagram of a SFME system. 
Amphi-solvent should be completely or partially miscible with both the immiscible fluids. SFMEs are to some extent similar to surfactant-based microemulsions (SBMEs). The first SFME was reported by Smith et al..$^{77}$ in 1977. It was a ternary system consisting of hexane, water and i-propanol. o/w as well as BC structures were observed. There are several ternary systems which have been found to form SFMEs. Some of these are: (i) toluene-water-propanol, (ii) benzene-water-propanol, (iii) benzene-water-ethanol, (iv) octanol-glycerol-ethanol and (v) perfume-water-ethanol. Surfactant-free emulsions are also possible but they are turbid and only kinetically stable. A recent example of SFME system comprising water/ethanol/dichloromethane showed three microregions - water-in-dichloromethane (w/o), bicontinuous (BC) and dichloromethane-in-water (o/w) regions $^{78}$. The amphi-solvent, ethanol serves the purpose of amphiphile which brings down IFT between water/ dichloromethane. Surfactants are generally costly and the amount of surfactants used in microemulsion formation is usually high. If ethanol can be used, then the preparation would be cost effective and may become useful in practice. Solid silica nanoparticles have been prepared in $\mathrm{o} / \mathrm{w}$ SFME. Two other SFMEs (water/1-propanol/limonene and water/tert-butanol/limonene) were studied recently to determine the activity of enzyme 'horseradish,79, where 1-propanol and tert-butanol served as the amphi-solvents. While comparing the triangular phase diagrams of various systems, it was observed that the monophasic part varied from about $5 \%$ to about $70 \%$ or more. However, it has not been possible to explain these results. It was also observed that temperature, $\mathrm{pH}$, ionic strength, etc. did not have much effect on monophasic region ${ }^{80-82}$. Further, if the oils are not long-chained hydrocarbons and fatty acid esters (vegetable oils, etc.), then normal surfactants may not always be necessary for the systems. Lower alkanols, termed as 'amphi-solvent', may serve emulsification process if the oils are like dichloromethane, chloroform, toluene, etc. As per literature report ${ }^{83}$, for coconut oil (a tough oil to disperse), a good stable microemulsion can be prepared employing ethanol and iso-propanol along with amphiphile Brij 52 when their proportion (Brij : alkanol) is $1: 1 \mathrm{w} / \mathrm{w})$. Different experimental methods like conductance, cyclic voltametry, NMR, DLS, SAXS, etc. are used in SFME studies. It needs to be stated here that the thermodynamic basis of SFME formation, microstructures, environmental factors, etc. are yet to be properly understood ${ }^{76}$. SFME formation of long-chain hydrocarbon oils is still a challenge in the field of surface science and technology.

A SFME consisting of isopentyl acetate (IA), n-propanol, and water, in which IA and $n$-propanol are used as oil phase and amphi-solvent respectively, has recently been reported ${ }^{84}$. Cyclic voltammetry, fluorescence spectroscopy and UV-visible spectroscopy techniques are used to determine microstructure and structural transitions.
Here, o/w, BC and w/o, phases were directly verified by cryo-TEM measurements. Composition change leads to structural transitions. This is, probably, the first time where, in SFME, a simple ester compound as the oil phase had been employed. A ternary detergentless microemulsion was obtained from water-octanol-ethanol system $^{85}$. Direct microstructural evidences were obtained by light, X-ray and neutron scattering studies. The stability of such microemulsion was explained by the proposition of existence of a balance between hydration force and entropy.

\section{Ionic liquid-based microemulsions}

In recent years, pollution, destruction of natural environment, etc. have become more important from political, industrial and academic points of view. Chemicals, particularly solvents are said to be the most important polluting agents. Hence there is an effort to replace organic solvents by something which will not have much damaging effect on nature ${ }^{86,87}$. Modern solvents like ionic liquids (ILs), particularly room temperature ionic liquids (RTILs): are quite often used as green substitute for polluting, environment damaging organic solvents ${ }^{88,89}$. ILs are also termed as 'designer solvents'. However, not all ILs are 'green solvents'. An interesting feature of ILs use is that they can be used as oil substitute, water substitute, co-surfactant as well as surfactants ${ }^{86}$. ILs are molten salts at room temperature containing ions. In general, salts which are liquid below $100^{\circ} \mathrm{C}$ are called 'ionic liquids'. Such salts were first reported in 1911 by Ray et al ${ }^{90-92}$. They were ethylammonium nitrite, trimethylammonium nitrite, tetramethylammonium nitrite, benzylammonium nitrite, etc. and were not very stable. In 1914, Walden first reported a stable salt of this type named ethylammonium nitrate ${ }^{93}$. It is interesting to note that the term 'ionic liquid' was first used in the scientific literature by Barrer $^{94}$ in 1943, the very year when first microemulsion work was published by Hoar and Schulman ${ }^{1}$. Barrer was born in New Zealand in 1910, studied at Cambridge, worked at various places including Imperial College, London, UK and died in London in 1996. Ionic liquids are used in the preparation of microemulsions as well as in various other aspects of surfactant science ${ }^{95}$. Probably the first polar core IL microemulsion reported by Gao et al. ${ }^{96}$ was $[\mathrm{bmim}]\left[\mathrm{BF}_{4}\right] / \mathrm{TX}-100 /$ cyclohexane system. Gao et $a l .{ }^{96}$ used freeze fracture electron microscopy (FFEM) for obtaining the size and shape of the aggregates in microemulsion. FFEM consists of: (1) freezing of the sample, (2) fracturing and replication, and (3) obtaining transmission electron microscopic (TEM) images. The weight fraction of TX-100 was kept constant at 0.45 and FFEMs were obtained of systems with IL to TX-100 ratios $(\mathrm{R})$ equal to $0.2,0.5,1.0$ and 1.5 respectively. It was found that as $\mathrm{R}$ increases, droplet sizes also increase (Figure 8).

CURRENT SCIENCE, VOL. 116, NO. 6, 25 MARCH 2019 
Seth and Sarkar ${ }^{88}$ prepared an IL microemulsion (IL/O) of composition $\left[\mathrm{bmim}^{2}\left[\mathrm{PF}_{6}\right] / \mathrm{TX}-100 /\right.$ water, where [bmim] $\left[\mathrm{PF}_{6}\right]$ is 1-butyl-3-methylimidazolium hexafluorophosphate and TX-100 is a non-ionic surfactant. They determined the hydrodynamic diameter of microemulsion as a function of composition by dynamic light scattering (DLS) measurements and found that the hydrodynamic diameter changes from 8.3 to 12.6 to $18.9 \mathrm{~nm}$ as the $\mathrm{wt} \%$ composition changes from $(83.9: 15.0: 1.1)$ to $(83.3: 15.1: 1.6)$ to $(81.5: 15.7: 2.8)$ as $\mathrm{H}_{2} \mathrm{O}: \mathrm{TX}-100$ : IL respectively. Pickart and Luczak ${ }^{97}$ studied various transport properties of aqueous IL microemulsions containing 1-butyl-3-methylimidazolium hexafluorophosphate, [bmim $]\left[\mathrm{PF}_{6}\right]$, and bis((trifluoromethanesulphonyl)imide, $[\mathrm{bmim}]\left[\mathrm{Tf}_{2} \mathrm{~N}\right]$ stabilized by the non-ionic surfactant TX100 , or its mixture with a co-surfactant, i.e. butanol. They observed that the presence of butanol increased conductivity and significantly decreased viscosity. The stronger the interaction between positive and negative ions of ILs, less was the conductivity in microemulsion. A schematic representation of IL comprising microemulsion is shown in Figure 9.

Zhang et al. ${ }^{98}$ discussed two non-aqueous IL microemulsions (toluene/TX-100/[bmim $]\left[\mathrm{PF}_{6}\right]$ and $[\mathrm{bmim}]$ $\left[\mathrm{BF}_{4}\right] / \mathrm{TX}-100 /$ benzene). They observed that the conductance of microemulsion is a function of IL concentration as well as the hydrophilicity of IL in the microemulsion.
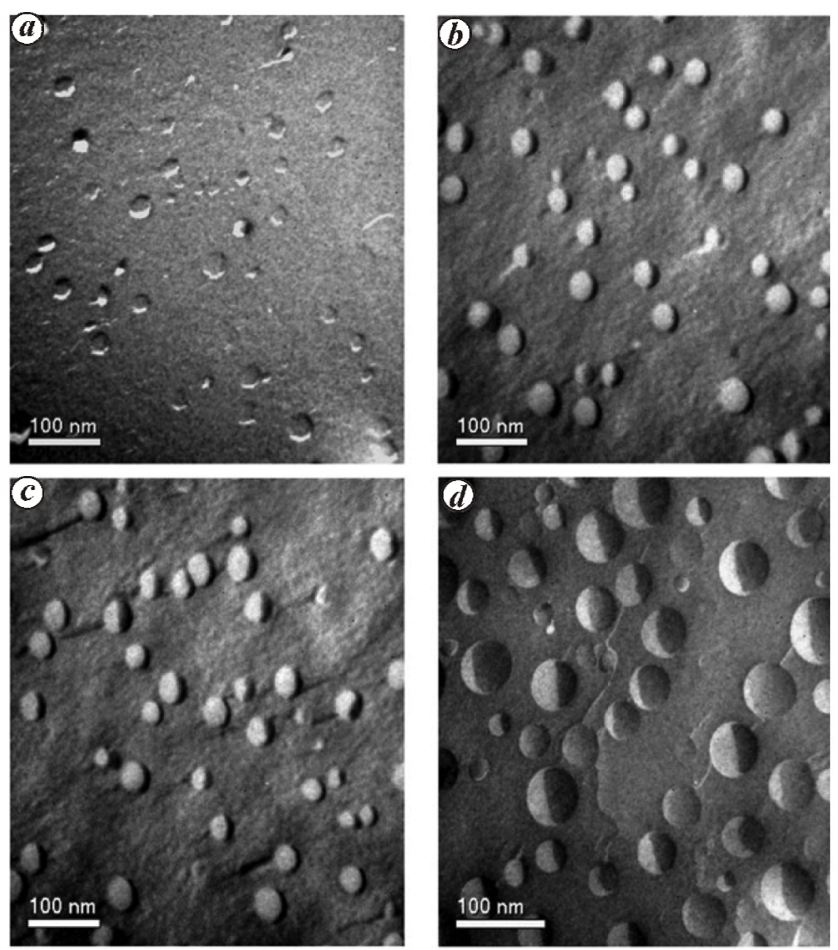

Figure 8. TEM images of FFEM replicas of $[\mathrm{bmim}]\left[\mathrm{BF}_{4}\right] / \mathrm{TX}$ 100 /cyclohexane microemulsion (TX-100 wt. fraction 0.45$) . R=0.2$, $0.5,1.0$ and 1.5 for $(\boldsymbol{a}),(\boldsymbol{b}),(\boldsymbol{c})$ and $(\boldsymbol{d})$ respectively. (Reproduced with permission of the Royal Society of Chemistry $)^{96}$.
According to them, dynamic percolation occurred in the toluene system, and in the benzene system it was static percolation. From dielectric spectroscopic study they suggested a possible coupling between TX-100 and IL.

Normally, ILs are polar, low melting and non-vapour forming. They may also be amphiphile like, surface active, form micelles and microemulsions. Surface active ILs are called SAIL. Solution and surface chemical behaviour of two phosphonium based ILs, triisobutyl (methyl) phosphonium tosylate (IL-1) and trihexyl (tetradecyl) phosphonium bis 2,4,4-(trimethylpentyl)phosphinate (IL-2) have been studied by Mukherjee et al. ${ }^{99}$. IL-1 can replace water in forming microemulsions with the oil isopropylmyristate (IPM), stabilized by IL-2 (surfactant) + isopropanol (IP) (co-surfactant), i.e. IL-1/IPM/ (IL-2+IP) system. It produces a large monophasic zone in the pseudo-ternary phase diagram. The droplet sizes and thermal stability of the formed IL-1/IPM microemulsion were determined. Stable microemulsion formation was also studied by others ${ }^{100,101}$. Rojas et al. ${ }^{102}$ studied microemulsions comprising IL, 1-ethyl-3-methylimidazolium $n$-hexylsulphate-water $(1: 1) /$ toluene-pentanol (1:1)/ SDS (CTAB). Moniruzzaman et al. ${ }^{103}$ studied IL(1-ethyl3-methylimidazolium tetrafluoroborate)-(polar phase)/ IPM-(oil) (Tween-80 + Span-20)-(surfactants) microemulsion, and found it to be a proficient vehicle to encapsulate different drugs. It may be added that zwitterionic, nonionic and anionic surfactants with hydrophobic IL (oil) are used to form o/w microemulsions ${ }^{104,105}$. From our study and other above studies, it may be noted that with ionic liquids, the following types of microemulsion can be formulated: (1) water/(SAIL + co-surfactant)/oil, (2) water/( $\left.\mathrm{SAIL}_{1}+\mathrm{SAIL}_{2}\right) /$ oil, (3) IL/surfactant + co-surfactant)/oil, (4) IL/(SAIL + co-surfactant)/oil, (5) IL/ $\left(\mathrm{SAIL}_{1}+\mathrm{SAIL}_{2}\right) /$ oil and $(6) \mathrm{IL} /\left(\mathrm{SAIL}_{1}+\mathrm{SAIL}_{2}\right) / \mathrm{IL}$ (oil like). Thus, we see that ILs may also form waterless microemulsion as mentioned earlier ${ }^{98}$. On the whole, presence of a co-surfactant may not be essential in the formation of microemulsion with IL as was the case with AOT or TX-100 based microemulsion mentioned earlier ${ }^{15-20}$; the phase diagrams are thus not essentially pseudoternary. This prospective field requires more exploration. Depending on their individual physicochemical properties they may find varied applications in
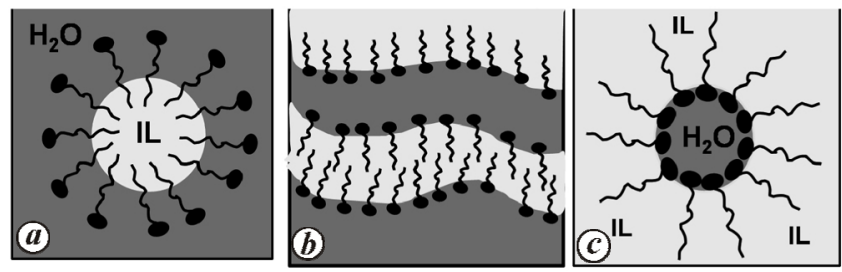

Figure 9. Schematic of microemulsion systems: $\boldsymbol{a}$, IL/water; $\boldsymbol{b}$, bicontinuous; $c$, water/IL. 
practice. Various techniques are used in the study of microstructure of IL based microemulsion. These are $\mathrm{SANS}^{103}, \mathrm{DLS}^{96}, \mathrm{SAXS}^{106}$, electrical conductivity measurement and so on. Addition of additives may affect the ternary phase diagram ${ }^{107-109}$ as is often seen in case of normal microemulsion. In most of the IL based nonaqueous microemulsions studies, the ILs consist of dialkylimidazolium cations with $\mathrm{BF}_{4}^{-}, \mathrm{PF}_{6}^{-}, \mathrm{NTf}_{2}^{-}$anions ${ }^{110}$. More information on IL behaviours in self aggregation processes is available in ref. 111.

\section{Non-aqueous microemulsion}

As mentioned earlier ${ }^{96}$, the first IL microemulsion was a non-aqueous one where water was replaced by the IL, $[\mathrm{bmim}]\left[\mathrm{BF}_{4}\right]$. The two other IL microemulsions mentioned earlier ${ }^{98}$ are also non-aqueous microemulsions. A recent IL based non-aqueous microemulsion where IL represented a polar phase was prepared ${ }^{112}$. Low-viscosity room-temperature ionic liquid (RTIL), 1-butyl-3-methylimidazolium bis(trifluoromethylsulphonyl)imide (bmim $\mathrm{Tf}_{2} \mathrm{~N}$ ), non-ionic surfactant Brij 30, and $n$-nonane were the components. The systems were characterized by DLS and SAXS; the IL/oil microemulsion was employed for the synthesis of gold nanoparticles from hydrogen tetrachloroaurate(III), $\mathrm{HAuCl}_{4}$, by $\mathrm{UV}$-photoreduction technique.

Several attempts were made to prepare and study waterless microemulsion. In these efforts water was replaced by polar non-aqueous solvents (like ethylene glycol, formamide, etc.); the preparations were essentially oil continuous. Friberg and Liang ${ }^{113}$ determined pseudoternary phase diagram of a four component system consisting of glycerol (GY), Na-dodecyl sulphate, hexanol and decane with various hexanol to decane weight ratios at $30^{\circ} \mathrm{C}$. They found only isotropic solutions and liquid crystalline phase. Diels-Alder reaction between $N$-ethylmaleimide and cyclopentadiene was studied in aqueous microemul$\operatorname{sion}^{114}$. It has been reported that Diels-Alder reaction was also studied in different microemulsion media ${ }^{115,116}$. Diels-Alder type reaction between $N$-ethylmaleimide and 2,3-dimethyl 1,3-butadiene was studied for the first time in an IL microemulsion. Rate constant, etc. were determined spectrophotometrically. The reaction was faster than that in isooctane or in an AOT aqueous microemul$\operatorname{sion}^{117}$. Ray and Moulik ${ }^{118}$ made a detailed study on systems comprising solvents like formamide (FA), ethylene glycol (EG), propylene glycol (PG), dimethyl formamide (DMF) and dimethyl acetamide (DMA), oils like heptane $(\mathrm{Hp})$, octane $(\mathrm{Oc})$, isooctane (i-Oc), xylene $(\mathrm{Xy})$ and toluene (Tl); and surfactant like AOT, sodium 1,4-bis(2ethylhexyl) sulphosuccinate. Ternary phase diagrams, conductance and viscosity of microemulsion systems were studied. Percolation of conductance and viscosity were observed supporting spherical or ellipsoidal non- solvated dispersions. The thermodynamics of solution of non-aqueous solvents in AOT/oil medium resulting in W IV microemulsion was determined from microcalorimetric measurements. Non-aqueous microemulsions containing AOT, $n$-hexane or $n$-heptane and water substitutes, like GY, EG, PG, FA, DMF and DMA were studied and compared with the corresponding aqueous systems. The solvatochromic behaviour of 1-methyl-8oxyquinolinium betaine (QB) was used to characterize the systems. In the case of GY, as well as for water, QB seems to be anchored at the interface and no partition was detected $^{119}$. Phase diagram of a non-aqueous microemulsion system containing cyclohexane/TX-100/DMF was determined at $40^{\circ} \mathrm{C}$. The one-phase microemulsion area increased in the presence of a co-surfactant. Various physical properties were studied and microstructures were determined. A cyclohexane/TX-100+1-propanol/ DMF system showed percolation, though not high, with ca. 0.15 mole fraction of DMF. Depending on the concentration of DMF, BC structure was found ${ }^{120}$. In Figure 10, a pseudo-ternary phase diagram of the system cyclohexane/CTAB + 1-propanol/DMF at different temperatures is presented $^{121}$. It was obvious that at higher temperatures the $1 \Phi$ microemulsion areas were larger and there were maxima. The effects of polymers like PEG 400 as well as PEG 4000 were also studied. Besides some changes in phase areas, no other changes were $\operatorname{seen}^{121}$. Ferreira et $a l .{ }^{122}$ suggested a glycerine-based microemulsion for enhanced oil recovery (EOR). The microemulsion used was comprised of commercial surfactants (DBB-7107 and DBB-7191), isopropyl alcohol, pine oil and glycerine for distilled water. Oil recovery was studied with samples of sandstone rock in a core holder. It was found that the recovery factor was almost $49 \%$ of the residual oil whereas it was about $36 \%$ of the residual oil in the corresponding aqueous microemulsion.

Fischer et al. ${ }^{123}$ studied surfactant-free and water-free green microemulsions glycerol/ethanol/1-octanol and

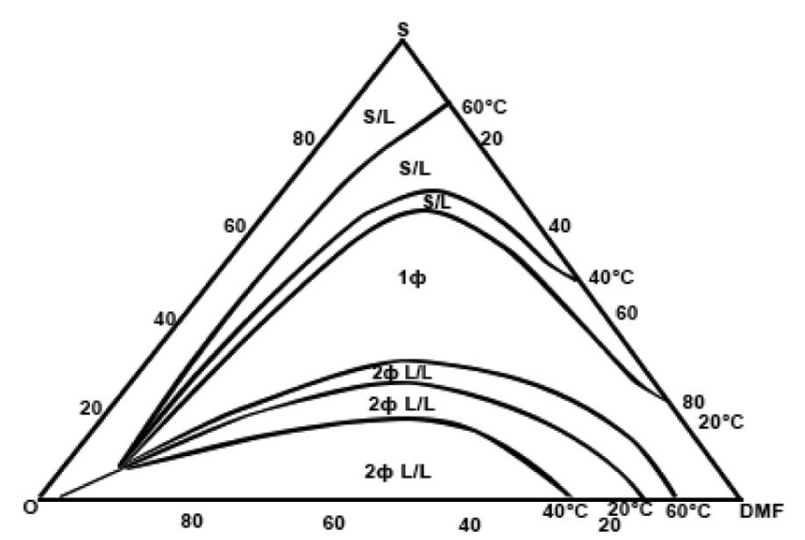

Figure 10. Pseudo-ternary phase diagram of system cyclohexane $(\mathrm{O}) /$ CTAB + 1-propanol $(1: 2) / \mathrm{DMF}$ at different temperatures ${ }^{121}$. 
deep eutectic solvent/tetrahydrofurfuryl alcohol/diethyl adipate to check the presence of nano-clustering. Ethylene glycol-choline chloride (molar ratio $4: 1$ ) and ureacholine chloride (molar ratio $2: 1$ ) were the deep eutectic solvents. Probably this was the first time such solvents were used to formulate microemulsions. Experimental methods such as phase diagrams, DLS and SAXS were used. Aggregate fluctuations were observed. The structures observed are similar to classical microemulsions. However, in classical systems, non-miscible phases have sharp boundary which were absent in these cases. Whether the acetonitrile/AOT $/ n$-heptane microemulsion, is a reverse micelle or $\mathrm{BC}$ microemulsion, is a controversial issue $^{124}$. The spectral and dynamical responses of a hydrophilic solvatochromic fluorophore 4-aminophthalimide (4-AP) at different $w_{\mathrm{s}}$ values (=[acetonitrile]/ [AOT]) has been used to answer the above question. The experimental results indicate that below $w_{\mathrm{s}}(\sim 1)$, the system is a reverse micelle, and above this, the system may remain as BC. In authors' words, 'The conjecture was further supported by dynamic light scattering measurements, where we observed a gradual increment of the average size at the low acetonitrile content (up to $w_{\mathrm{s}}=1$ ) but, thereafter, the size distribution becomes multimodal and sizes cannot be estimated correctly ${ }^{124}$.

\section{Uses and applications of microemulsions}

Microemulsions have numerous uses and applications, like solubilization, cleaning, fuels, tertiary oil recovery, cosmetics, pharmaceutics, corrosion inhibitors, drug encapsulation and delivery, templates for nano material preparation, membrane filtration, detoxification, etc. A comprehensive information and knowledge on this subject can be found in the review of Paul and Moulik ${ }^{24}$, and Moulik and Rakshit ${ }^{23}$ and references therein. w/o and $\mathrm{o} / \mathrm{w}$ systems are used depending on the necessity. The needs of different types of microemulsions are on the rise due to advancement of research in this field. In current situation, foremost applications of microemulsions are in cosmetics, nano-structured material synthesis and drug encapsulation and delivery. Demands for cream, body wash, shampoo, lotions are always on the rise; for electronic and digitization gadgets nano metal oxides, sulphides, selenides, carbon nanotubes, etc. are in great demand; drug encapsulation in microemulsion capsules and their release in the targeted location are in rapid rise in the fields of pharmacy and medicine. The study gives importance for easy preparation of varied types of microemulsions, and their good stability. Micelles, liposomes, vesicles, gels, coacervates are usable templates for the preparation of nanomaterials but they have a drawback of loose and ill defined shapes and instability. Materials encapsulated in them may easily leak or diffuse out. Materials formed and encapsulated in microemulsion droplets are less prone to diffusing and have more or less globular shape (although there are variations) with lower degree of heterogeneity. Therefore, preparation of globular and near globular materials is feasible using microemulsions. Most drugs which have potential uses are hydrophobic in nature; they can be dispersed and protected in $\mathrm{o} / \mathrm{w}$ microemulsions and are compatible with the environment of the body fluid. Catalysts, semiconductors, etc. are mostly inorganic materials and water insoluble. Thus, they are prepared in w/o microemulsion by suitable chemical processes ${ }^{21-27,125-127}$. They remain embedded in the water pool from where they are isolated and stored in congenial environments. There are several citations in the literature on the preparation of these materials and their properties. A few of them are cited here. For drug encapsulation and uses, bio-friendly systems are required. We present here a couple of references related to drug encapsulation and delivery ${ }^{128-130}$. These dispersions have various cosmetic applications because of small droplet size with high interfacial area, transparent or translucent appearance, high solubilization capacity, low viscosity, etc.

Microemulsions are good delivery systems for transportation of lipophilic compounds because they help in skin penetration which is important in cosmetic product formulations. It is used as antiperspirant, as body gel, sunscreen lotion as well as in hair care products. The importance of its use as cosmetics is because of higher stability and increased efficiency, and capability of solubilizing both hydrophilic and lipophilic ingredients of a cosmetic formulation thereby increasing drastically the spectrum of useable ingredients ${ }^{131,132}$. Halloran and Hoag ${ }^{133}$ used silicon oil and cationic surfactant microemulsion which has been found useful in hair combing. Similarly, von Rybinski et al. ${ }^{134}$ formulated clear, freeze stable hair conditioner. Fragrance is somewhat associated with the rate of evaporation of the system. Microemulsion seems to affect the rate of evaporation of limonene ${ }^{135}$. Friberg $^{136}$ studied the vapour pressure of phenethyl alcohol and phenethyl acetate from a microemulsion system and showed that the vapour pressure was clearly dependent upon the mole fraction of the perfume in the solution. Further, Kim et al. ${ }^{137}$ showed that w/o microemulsion can be used for removal of oily make up cosmetics. Here wet-ability and low viscosity are two important factors. Microemulsions have free flow and adhere to the treated area without tackiness. The ingredients include didecanoyl glycerol to increase melanin content, vitamin $\mathrm{E}$ for moisturizing effect, tri-decyl salicylic acid as anti-ageing agent. Benzotriazoles, bisesorecinyl triazine and S-triazine are added in microemulsion for photoprotective efficacy. Microemulsion containing ascorbyl palmitate effectively prevents UV-A-induced lipid peroxidation ${ }^{138}$. Anthocyanins are natural antioxidants present in nature. Anthocyanin microemulsion has been found to be w/o type, and it increases the stability and fat solubility ${ }^{139}$. 


\section{REVIEW ARTICLE}

Microemulsions are used in detergents. This is a complicated science because of the presence of surfaces and soils. For example, generally inorganic salts, sugar, urea, etc. are water soluble whereas fats, carbohydrates, proteins, etc. are not. Soil can be any of these or even a combination of all these. Organic dyes, pigments, etc. are also part of it. Surfaces may be hard, e.g. glass, steel, ceramics, etc. There are also soft surfaces like wool, polymer, cotton, etc. There are surfaces which cannot be washed with water and one uses non-aqueous microemulsion for this purpose. Microemulsions are most often used to remove oily soils. A heavy duty detergent formulation contains surfactants and builders to the extent of around $20 \%$ to $50 \%$ or more. Rest of the formulation is mostly water though fragrance, optical brighteners, cobuilders, soil repellents, etc. are also present in very small amounts. Generally in situ microemulsions are formed where soil behaves as an oil phase. In a typical working solution, surfactant concentration is $3-10$ times the critical micelle concentration of the surfactant $t^{134}$. An o/w microemulsion as detergent with linear medium chain fatty alcohols (C5-C8) as the oil phase, sodium dodecyl sulfate (SDS) and isomeric alcohol ethoxylate $\left(\mathrm{IC}_{13} \mathrm{EO}_{6}\right)$ as a mixed surfactant, and 1-butanol as co-surfactant was studied and the effect of addition of water was noted. It was found that with addition of water, microemulsion structure changed from w/o to $\mathrm{BC}$ to o/w. Even after 500 times dilution, the microemulsion detergency was more than that of standard liquid detergents ${ }^{140}$. The performance study of a mixture of sodium dioctyl sulphosuccinate, alkyl diphenyl oxide disulphonate, and sorbitan monooleate was done with hydrophobic oils; hexadecane and motor oil. The maximum detergency was observed for a W III microemulsion and also for a W I microemulsion. It is suggested that microemulsion formation and the resulting IFT reduction are important mechanisms in oily soil detergency ${ }^{141}$.

Microemulsion is also used as fuel. Biosurfactant mixtures are used in vegetable oil extraction for biofuel application. Reverse-micellar microemulsions of diesel are used. Out of the three Winsor types of microemulsions for the biosurfactant mixture of lecithin, rhamnolipid and sophorolipid with diesel at relatively low salinity, only W II microemulsions are suitable for vegetable oil extraction process as it obeys 'like-dissolveslike' principle. This microemulsion is more efficient in extracting vegetable oil than diesel or even hexane ${ }^{142}$. Water-in-diesel microemulsion and emulsion were prepared and their combustion characteristics, such as flame temperature, soot concentration, combustion duration, etc. were compared. In almost all properties, water-indiesel systems appeared to behave better than the diesel itself $^{143}$. A microemulsion consisting of coconut oil, aqueous ethanol, a surfactant (butane-1-ol) was prepared and tested as diesel engine fuel. It was found that the efficiency of this fuel was comparable to that of diesel.
Moreover, the components are renewable in nature and are environment friendly ${ }^{144}$.

\section{Summary}

Microemulsion was an important discovery in the mid 1940s though some microemulsion-type systems were already in the market at that time. These commercial systems were developed by trial and error methods and not by any scientific knowledge or insight. The 1943 paper in Nature by Hoar and Schulman is the first scientific study of microemulsion and we are now at the 75th year of that famous publication. This review article is to commemorate the elegance and importance of that 1943 paper on microemulsion formation. In this review we have discussed various aspects of the system including thermodynamic reasons of its formation and stability, fascinating internal complex structures, several important properties, and numerous practical applications. The progress of microemulsion research is still significantly visible.

1. Hoar, T. P. and Schulman, J. H., Transparent water-in-oil dispersions: the oleopathic hydro-micelle. Nature, 1943, 152, 102-103.

2. Schulman, J. H., Stoeckenius, W. and Prince, L. M., Mechanism of formation and structure of microemulsions by electron microscopy. J. Phys. Chem., 1959, 63, 1677-1680.

3. Bowcot, J. E. and Schulman, J. H., Surfactants. Z. Electrochem., 1955, 59, 283-287.

4. Prince, L. M. (ed.), Microemulsions: Theory and Practice, Academic Press, New York, 1977.

5. Prince, L. M., In Emulsion and Emulsion Technology-Part I, Surfactant Science Series (ed. Lissant, K. J.), Mercel Dekker, New York, 1974, vol. 6.

6. Moulik, S. P. and Mukherjee, K., On the versatile surfactant Aerosol OT (AOT): Its physicochemical and surface chemical behaviours and uses. Proc. Ind. Natl. Sci. Acad., 1996, 62A, 215232.

7. Tadros, Th. F., Surfactants, Academic Press, London, 1984.

8. Prince, L. M. J., A theory of aqueous emulsions I. Negative interfacial tension at the oil/water interface. J. Colloid Interf. Sci., 1967, 23, 165-173.

9. Rosoff, M., In Progress in Surface and Membrane Science (eds Cadenhead, D. A. and Danielli, J. F.), Academic Press, New York, 1978, vol. 12, p. 419.

10. Kaptay, G., On the negative surface tension of solutions and on spontaneous emulsification. Langmuir, 2017, 33, 10550-10560.

11. Bialke, J., Siebert, J. T., Lowen, H. and Speck, T., Negative interfacial tension in phase-separated active Brownian particles. Phys. Rev. Lett., 2015, 115, 098301(1-5).

12. Patashinski, A. Z., Orlik, R., Paclawski, K., Ratner, M. A. and Grzybowski, B. A., The unstable and expanding interface between reacting liquids: theoretical interpretation of negative surface tension. Soft Mater., 2012, 8, 1601-1608.

13. Ress, G. D. and Robinson, B. H., Microemulsions and organogels: properties and novel applications. Adv. Mater., 1993, 5, 608-619.

14. Pileni, M. P., Chandler, R. R., Gutsche, D. C., Alam, I., Pinizzoto, R. F. and Yang, H., An examination of the interfacial interactions between quantum-confined cadmium sulfide clusters and aminocalixarene stabilizer molecules. J. Phys. Chem., 1993, 97, 696-702. 
15. Rakshit, A. K., Livingstone, N. C. S. and Palepu, R. M., Physiochemical properties of water/AOT/isooctane microemulsion system in the presence of pyrrolidine derivatives and polyvinyl pyrrolidone polymers. J. Mol. Liq., 2003, 107, 235-256.

16. Fanun, M. (ed.), Microemulsions Properties and Applications, Surfactant Science Series 144, CRC Press, Boca Raton, USA, 2009, chap. 5.

17. Nave, S., Eastoe, J., Heenan, R. K., Steytler, D. and Grillo, I., What is so special about aerosol-OT? 2. Microemulsion systems. Langmuir, 2000, 16, 8741-8748.

18. Cho, Y. H., Kim, S., Bae, E. K., Mok, C. H. and Park, J., Formulation of a cosurfactant-free $\mathrm{O} / \mathrm{W}$ microemulsion using non-ionic surfactant mixture. J. Food Sci., 2008, 73, E115-E121.

19. Tessy, E. I. and Rakshit, A. K., Studies of cyclohexane/TX $100+$ ethylpropionate/water system: effect of ethylpropionate as cosurfactant. Colloid Polym. Sci., 1997, 275, 298-302.

20. Zhu, D. M., Wu, X. and Schelly, Z. A., Reverse micelles and water in oil microemulsions of Triton X 100 in mixed solvents of benzene and $n$-hexane. Dynamic light scattering and turbidity studies. Langmuir, 1992, 8, 1538-1540.

21. Moulik, S. P., Rakshit, A. K. and Capek, I., Microemulsions as templates for nanomaterials. In Microemulsions (ed. Stubenrauch, C.), Wiley, Chichester, UK, 2009, chap. 6, pp. 180-210.

22. Rakshit, A. K. and Moulik, S. P., Physicochemistry of W/O microemulsions: formation, stability and droplet clustering. In Microemulsions (ed. Fanun, M.), Surfactant Science Series 144, CRC Press, Boca Raton, USA, 2009, chap. 2, pp. 17-57.

23. Moulik, S. P. and Rakshit, A. K., Physicochemistry and applications of microemulsions. J. Surf. Sci. Technol., 2006, 22, 159186.

24. Paul, B. K. and Moulik, S. P., Uses and applications of microemulsions. Curr. Sci., 2001, 80, 990-100.

25. König, R. Y. G. and Stubenrauch, C., Pt nanoparticles via oil-inwater microemulsions stabilized by a technical grade surfactant: An economical and ecological approach. Tenside Surf. Det., 2015, 52, 106-112.

26. Sanchez-Dominguez, M. and Boutonnet, M., Synthesis of nanostructured catalytic materials from microemulsions. Catalysts 2016, 6, 4-8 and references therein.

27. Youssry, M., Guyomard, D. and Lestriez, B., Carbon black dispersions in surfactant based microemulsions. J. Mater. Res., 2018, 33, 1301-1307.

28. Winsor, P. A., Hydrotropy, solubilisation and related emulsification processes. Trans. Faraday Soc., 1948, 44, 376-398.

29. Winsor, P. A., Binary and multicomponent solutions of amphiphilic compounds. Solubilization and the formation, structure, and theoretical significance of liquid crystalline solutions. Chem. Rev., 1968, 68, 1-40.

30. John, A. C. and Rakshit, A. K., Formation of microemulsion: effect of temperature and polyacrylamide. J. Colloid Interf. Sci. 1993, 156, 202-206.

31. Bancroft, W. D., The theory of emulsification, V. J. Phys. Chem., 1913, 17, 501-519.

32. Bancroft, W. D., The theory of emulsification, VI. J. Phys. Chem., 1915, 19, 275-309.

33. Griffin, W. C., Classification of surface-active agents by 'HLB' J. Soc. Cosmet. Chem., 1949, 1, 311-326.

34. Griffin, W. C., Calculation of HLB values of non-ionic surfactants. J. Soc. Cosmet. Chem., 1954, 5, 249-256.

35. Davies, J. T., Proceedings of the Second International Congress of Surface Activity, Butterworths, London, 1957, vol. 1, p. 426.

36. Adamson, A. W., Physical Chemistry of Surfaces, John Wiley, New York, 1976.

37. Winsor, P. A., Solvent Properties of Amphiphilic Compounds, Butterworths, London, 1954.

38. Bourrel, M. and Schechter, R. S., Microemulsions and Related Systems, Mercel Dekker, New York, 1988.
39. Salager, J. L., Anton, R., Forgiarini A. and Marquez, L., In Microemulsions (ed. Stubenrauch, C.), Wiley, Chichester, UK, 2009, chap. 3, pp. 84-121.

40. Giustini, M., Murgia, S. and Palazzo, G., Does the Schulman's titration of microemulsions really provide meaningful parameters? Langmuir, 2004, 20, 7381-7384.

41. Burauer, S., Sachert, T., Sottmann, T. and Strey, R., On microemulsion phase behavior and the monomeric solubility of surfactant. Phys. Chem. Chem. Phys., 1999, 1, 4299-4306.

42. Paroor, H. M., Microemulsion: Prediction of the phase diagram with a modified Helfrich free energy. A Ph D dissertation, Johannes Gutenberg University, Mainz, Germany, 2012.

43. Vollmer, D., How to calculate phase diagrams for microemulsions - a simple rule. Fett-/Lipid, 1999, 101, 379-388.

44. Graciaa, A., Lachaise, J., Cucuphat, C., Bourrel, M. and Salager, J. L., Improving solubilization in microemulsions with additives. 1. The lipophilic linker role. Langmuir, 1993, 9, 669-672.

45. Graciaa, A., Lachaise, J., Cucuphat, C., Bourrel, M. and Salager, J. L., Improving solubilization in microemulsions with additives. 2. Long chain alcohols as lipophilic linkers. Langmuir, 1993, 9, 3371-3374.

46. Acosta, E., Uchiyama, H., Sabatini, D. and Harwell, J. H., The role of hrdrophilic linkers. J. Surfactants Deterg., 2002, 5, 151157.

47. Jakobs, B., Sottmann, T., Strey, R., Allgaier, J., Willner L. and Richter, D., Amphiphilic block copolymers as efficiency boosters for microemulsions. Langmuir, 1999, 15, 6707-6711.

48. Bisal, S., Bhattacharya, P. K. and Moulik, S. P., Conductivity study of microemulsions: dependence of structural behavior of water/oil systems on surfactant, cosurfactant, oil, and temperature. J. Phys. Chem., 1990, 94, 350-355.

49. Tjaden, B., Cooper, S. J., Brett, D. J. L., Kramer, D. and Shearing, P. R., On the origin and application of the Bruggeman correlation for analysing transport phenomena in electrochemical systems. Curr. Opin. Chem. Eng., 2016, 12, 44-51.

50. Bisal, S., Bhattacharya, P. K. and Moulik, S. P., Conductivity study of microemulsions: evaluation of hydration of oil/water microemulsions applying Bruggeman equation. J. Phys. Chem., 1990, 94, 4212-4216.

51. Paul, S., Bisal, S. and Moulik, S. P., Physicochemical studies on microemulsions: test of the theories of percolation. J. Phys. Chem., 1992, 96, 896-901.

52. Moulik, S. P. and Pal, B. K., Structure, dynamics and transport properties of microemulsions. Adv. Colloid Interf. Sci., 1998, 78, 99-195.

53. Pithapurwala, Y. K. and Shah, D. O., Interfacial composition of microemulsions: modified schulman-bowcott model. Chem. Eng. Commun., 1984, 29, 101-112.

54. Wang, Y., Ph D thesis, Massey University, Auckland, New Zealand, 2014.

55. Birdi, K. S., Microemulsions: effect of alkyl chain length of alcohol and alkane. Colloid Polym. Sci., 1982, 260, 628-631.

56. De, M., Bhattacharya, S. C., Moulik, S. P. and Panda, A. K., Interfacial composition, structural and thermodynamic parameters of eater/(surfactant $+n$-butanol) $/ n$-heptane water-in-oil microemulsion formation in relation to the surfactant chain length. J. Surfact. Deterg., 2010, 13, 475-484.

57. Moulik, S. P., Digout, L. G., Aylward, W. M. and Palepu, R., Studies on the interfacial composition and thermodynamic properties of W/O microemulsions. Langmuir, 2000, 16, 31013106 .

58. Fang, J. and Venable, R. L., Conductivity study of the microemulsion system sodium dodecyl sulfate-hexylamine-heptanewater. J. Colloid Interf. Sci., 1987, 116, 269-277.

59. Mehta, S. K., Kaur, K., Kaur, G. and Bhasin, K. K., Microemulsion, Properties and Applications (ed. Fanun, M.), Taylor \& Francis, USA, 2009, chap. 3. 
60. Eicke, H. F., Borkovec, M. and Gupta, B. D., Conductivity of water-in-oil microemulsions: a quantitative charge fluctuation model. J. Phys. Chem., 1989, 93, 314-317.

61. Hait, S. K., Sanyal, A. and Moulik, S. P., Physicochemical studies on microemulsions. 8. The effects of aromatic methoxy hydrotropes on droplet clustering and understanding of the dynamics of conductance percolation in water/oil microemulsion systems. J. Phys. Chem. B, 2002, 106, 12642-12650.

62. Dogra, A. and Rakshit, A. K., Phase behavior and percolation studies on microemulsion system water/SDS + Myrj45/cyclohexane in the presence of various alcohols as cosurfactants. J. Phys. Chem. B, 2004, 108, 10053-10061.

63. Lagues, M., Ober, R. and Taupin, C., Study of structure and electrical conductivity in microemulsions: Evidence for percolation mechanism and phase inversion. J. Phys. Lett., 1978, 39, L487-L491.

64. Lagues, M. and Sauterey, C., Percolation transition in water in oil microemulsions. Electrical conductivity measurements. J. Phys. Chem., 1980, 84, 3503-3508.

65. Paul, B. K., Mitra, R. and Moulik, S. P., Microemulsions: Percolation of conductance and thermodynamics of droplet clustering. In Encyclopedia of Surface and Colloid Science (ed. Somasundaran, P.), Marcel Dekker Inc., Taylor \& Francis, USA, 2006, pp. 3927-3956.

66. Alexandradis, P., Holzwarth, J. F. and Hatton, T. A., Thermodynamics of droplet clustering in percolating AOT water-in-oil microemulsions. J. Phys. Chem., 1995, 99, 8222-8232.

67. Paul, B. K. and Moulik, S. P., Biological microemulsions: Part III - the formation characteristics and transport properties of saffola-aerosol OT-hexylamine-water system. Indian J. Biochem. Biophys., 1991, 28, 174-183.

68. Mukherjee, K., Mukherjee, D. C. and Moulik, S. P., Thermodynamics of microemulsion formation. J. Colloid Interf. Sci., 1997, 187, 327-333.

69. Ajith, S., John, A. C. and Rakshit, A. K., Physicochemical studies of microemulsions. Pure Appl. Chem., 1994, 66, 509-514.

70. John, A. C. and Rakshit, A. K., Phase behavior and properties of a microemulsion in the presence of $\mathrm{NaCl}$. Langmuir, 1994, 10, 2084-2087.

71. Santhanalakshmi, J. and Parameswari, A., Microstructure and dynamics of water-in-oil microemulsion. Indian J. Chem., 1992, 31A, 630-638.

72. Chen, S. J., Evans, D. F. and Ninham, B. W., Properties and structure of three-component ionic microemulsions. J. Phys. Chem., 1984, 88, 1631-1634.

73. Katchalsky, A. and Curran, P. F., Nonequilibrium Thermodynamics in Biophysics, Harvard University Press, Cambridge, USA, 1967, chap. 8, p. 88.

74. Ray, S., Biswal, S. R. and Moulik, S. P., Studies on structure and dynamics of microemulsions II: viscosity behaviour of water-inoil microemulsions. J. Surf. Sci. Technol., 1992, 8, 191-199.

75. Joshi, A. L. and Rakshit, A. K., Physicochemical studies of cyclohexane/CTAB/1-propanol/water microemulsion system in the presence of PEG 400. Indian J. Chem., 1997, 36A, 38-45.

76. Hou, W. and Xu, J., Surfactant-free microemulsions. Curr. Opin. Coll. Interf. Sci., 2016, 25, 67-74.

77. Smith, G. D., Donelan, C. E. and Barden, R. E., Oil-continuous microemulsions composed of hexane, water, and 2-propanol. J. Colloid Interf. Sci., 1977, 60, 488-496.

78. Sun, B., Chai, J., Chai, Z., Zhang, X., Cui, X. and Lu, J., A surfactant-free microemulsion consisting of water, ethanol, and dichloromethane and its template effect for silica synthesis. J. Colloid Interf. Sci., 2018, 526, 9-17.

79. Krick, S., Touraud, D., Bauduin, P., Zinn, T. and Kunz, W., Enzyme activity of horseradish peroxidase in surfactant-free microemulsions. J. Colloid Interf. Sci., 2018, 516, 466-475.

80. Lund, G. and Holt, S., Detergentless water/oil microemulsions: IV. The ternary pseudo-phase diagram for and properties of the system toluene/2-propanol/water. J. Am. Oil Chem. Soc., 1980, 57, 264-267.

81. Kelsar, B. A., Veric, D., Barden, R. E. and Holt, S. L., Detergentless water/oil microemulsions composed of hexane, water, and 2 propanol. 2. Nuclear magnetic resonance studies, effect of added sodium chloride. J. Phys. Chem., 1979, 83, 1276-1280.

82. Lara, J., Perron, G. and Desnoyers, J. E., Heat capacities and volumes of the ternary system benzene-water-2-propanol. $J$. Phys. Chem., 1981, 85, 1600-1605.

83. Acharya, A., Moulik, S. P., Sanyal, S. K., Mishra, B. K. and Puri, P. M., Physicochemical investigations of microemulsification of coconut oil and water using polyoxyethylene 2-cetyl ether (Brij 52) and isopropanol or ethanol. J. Colloid Interf. Sci., 2002, 245, 163-170.

84. Liu, Y., Xu, J., Deng, H., Song, J. and Hou, W., A surfactant-free microemulsion composed of isopentyl acetate, $n$-propanol, and water. RSC Adv., 2018, 8, 1371-1377.

85. Zemb, T. N. et al., How to explain microemulsions formed by solvent mixtures without conventional surfactants. Proc. Natl. Acad. Sci., 2016, 113, 4260-4265.

86. Mehta, S. K. and Kaur, K., Ionic liquid microemulsions and their technological applications. Indian J. Chem., 2010, 49A, 662-684.

87. Vashishat, R., Chabba, S. and Mahajan, R. K., Surface active ionic liquid induced conformational transition in aqueous medium of hemoglobin. RSC Adv., 2017, 7, 13041-13052.

88. Seth, D. and Sarkar, N., In Microemulsions Properties and Applications, Surfactant Science Series (ed. Fanun, M.), CRC Press, Boca Raton, USA, 2009, vol. 144.

89. Kuchlyan, J., Kundu, N. and Sarkar, N., Ionic liquids in microemulsions: formulation and characterization. Curr. Opinion Coll. Surf. Sci., 2016, 25, 27-38.

90. Ray, P. C. and Sen, H. K., CLXVI - tetramethylammonium hyponitrite and its decomposition by heat. J. Chem. Soc., 1911, 99, 1466-1470.

91. Ray, P. C. and Rakshit, J. N., CLXVII - nitrites of the alkylammonium bases: ethylammonium nitrite, dimethylammonium nitrite, and trimethylammonium nitrite. J. Chem. Soc., 1911, 99, 1470-1475.

92. Ray, P. C. and Datta, R. L., CLXVIII - nitrites of the benzylammonium series. Benzylammonium nitrite and dibenzylammonium nitrite and their sublimation and decomposition by heat. J. Chem. Soc., 1911, 99, 1475-1477.

93. Walden, P., Molecular weights and electrical conductivity of several fused salts. Bull. Acad. Imper. Sci. (St Petersburg), 1914, 1800, 405-422.

94. Barrer, R. M., The viscosity of pure liquids. II. Polymerised ionic melts. Trans. Faraday Soc., 1943, 39, 59-67.

95. Mukherjee, I. and Moulik, S. P., Ionic liquids in colloidal regime. In Ionic Liquid-Based Surfactant Science: Formulation, Characterization, and Applications (eds Paul, B. K. and Moulik, S. P.), Wiley, Hoboken, New Jersey, 2015, chap. 10, 1st edn.

96. Gao, H., Li, J., Han, B., Chen, W., Zhang, J., Zhang, R. and Yan, D., Microemulsions with ionic liquid polar domains. Phys. Chem. Chem. Phys., 2004, 6, 2914-2916.

97. Piekart, J. and Luczak, J., Transport properties of aqueous ionic liquid microemulsions: influence of the anion type and presence of the cosurfactant. Soft Matter, 2015, 11, 8992-9008.

98. Zhang, C., Zhen, Z., Ma, L. and Zhao, K., Dielectric relaxation of nonaqueous ionic liquid microemulsions: polarization, microstructure, and phase transition. RSC Adv., 2017, 7, 13733-13741.

99. Mukherjee, I., Mukherjee, S., Naskar, B., Ghosh, S. and Moulik, S. P., Amphiphilic behavior of two phosphonium based ionic liquids. J. Colloid Interf. Sci., 2013, 395, 135-141.

100. Atkin, R. and Warr, G. G., Phase behavior and microstructure of microemulsions with a room-temperature ionic liquid as the polar phase. J. Phys. Chem. B, 2007, 111, 9309-9316. 
101. Eastoe, J., Gold, S., Rogers, S. E., Paul, A., Welton, T., Heenan, R. K. and Grillo, I., Ionic liquid-in-oil microemulsions. J. Am. Chem. Soc., 2005, 127, 7302-7303.

102. Rojas, O., Tiersch, B., Frasca, S., Wollenberger, U. and Koetz, J., A new type of microemulsion consisting of two halogen-free ionic liquids and one oil component. Colloid Surface A, 2010, 369, 82-87.

103. Moniruzzaman, M., Kamiya, N. and Gato, M., Ionic liquid based microemulsion with pharmaceutically accepted components: Formulation and potential applications. J. Colloid Interf. Sci., 2010, 352, 136-142.

104. Behara, K., Kumar, V. and Pandey, S., A novel water-in-ionic liquid microemulsion and its interfacial effect on the activity of laccase. Colloid Surface B, 2011, 82, 432-437.

105. Paroda, J. H., Mansueto, M., Laochat, S. and Stubenrauch, C., Microemulsions with novel hydrophobic ionic liquids. Soft Matter, 2011, 7, 6805-6810.

106. Li, J., Zhang, J., Gao, H., Han, B. and Gao, I., Nonaqueous microemulsion-containing ionic liquid $[\mathrm{bmim}]\left[\mathrm{PF}_{6}\right]$ as polar microenvironment. Colloid Polym. Sci., 2005, 283, 1371-1375.

107. Gao, Y., Hilfert, L., Voigt, A. and Sundmacher, K., Decrease of droplet size of the reverse microemulsion 1-butyl-3-methylimidazolium tetrafluoroborate/Triton X-100/cyclohexane by addition of water. J. Phys. Chem. B, 2008, 112, 3711-3719.

108. Cheng, S., Han, F., Wang, Y. and Yan, J., Effect of cosurfactant on ionic liquid solubilization capacity in cyclohexane/TX-100/1butyl-3-methylimidazolium tetrafluoroborate microemulsions. Colloid Surface A, 2008, 317, 457-461.

109. Li, N., Zhang, S., Li, X., Yu, C. and Zhang, L., Effect of polyethylene glycol (PEG-400) on the 1-butyl-3-methylimidazolium tetrafluoroborate-in-cyclohexane ionic liquid microemulsion. Colloid Polym. Sci., 2009, 287, 103-108.

110. Ren, Q., Yang, Q., Su, B., Zhang, Z., Bao, Z. and Xing, H., Ionic liquid based nonaqueous microemulsion. In Ionic Liquid-based Surfactant Science: Formulation, Characterization and Applications, Wiley Series on Surface and Interfacial Chemistry, Wiley, New Jersey, USA, 2015, chapter 17, pp. 43-359.

111. Pal, B. K and Moulik, S. P., Ionic Liquid-based Surfactant Science: Formulation, Wiley Series on Surface and Interfacial Chemistry, Wiley, New Jersey, USA, 2015.

112. Mamusa, M., Arroyo, M. C., Fratini, E., Giorgi, R. and Baglioni, P., Nonaqueous microemulsion in the Bmim Tf2N/Brij 30/nnonane system: Structural investigation and application as gold nanoparticle microreactor. Langmuir, 2018, 34, 12609-12618; doi:10.1021/acs.langmuir8b02420.

113. Friberg, S. E. and Liang, Y. C., Critical phenomena in nonaqueous microemulsions. Colloid Surface, 1987, 24, 325-336.

114. Engberts, J. B. F. N., Fernandez, E., Garcia-Rio, L. and Leis, J. R., Water in oil microemulsions as reaction media for a DielsAlder reaction between $N$-ethylmaleimide and cyclopentadiene. J. Org. Chem., 2006, 71, 4111-4117.

115. Holmberg, K., Lassen, B. and Stark, M. B., Enzymatic glycerolysis of a triglyceride in aqueous and nonaqueous microemulsions. J. Am. Oil Chem. Soc., 1989, 66, 1796-1800.

116. Lattes, A. and Rico, I., Aggregation in formamide solution: Reactivity and structure of non-aqueous microemulsions. Colloid Surface, 1989, 35, 221-235.

117. Lu, H., An, X., Yu, J. and Song, X., Diels-Alder reaction in microemulsions with ionic liquid. J. Phys. Org. Chem., 2012, 25, $1210-1216$.

118. Ray, S. and Moulik, S. P., Dynamics and thermodynamics of aerosol OT-aided nonaqueous microemulsions. Langmuir, 1994, 10, 2511-2515.

119. Falcone, R. D., Correa, N. M., Biasutti, M. A. and Silber, J. J., Properties of AOT aqueous and nonaqueous microemulsions sensed by optical molecular probes. Langmuir, 2000, 16, 30703076 .
120. Tessy, E. I. and Rakshit, A. K., The physicochemical properties of a nonaqueous microemulsion: cyclohexane/Triton ${ }^{\circledR} \mathrm{X}-100 /$ dimethylformamide at $40^{\circ}$ C. Bull. Chem. Soc. Jpn, 1995, 68, 2137-2141.

121. Joshi, A. L., Ph D thesis. M.S. University of Baroda, Vadodara, Gujarat, India, 1999.

122. Ferreira, G. F. D., Souza, D. R. Q., Lima, R., Lobato, A. K. C. L., Silva, A. C. M. and Santos, L. C. L., Novel glycerin-based microemulsion formulation for enhanced oil recovery. J. Petrol. Sci. Eng., 2018, 167, 674-681.

123. Fischer, V., Marcus, J., Jouraud, D., Diat, O. and Kunz, W., Toward surfactant-free and water-free microemulsions, J. Colloid Interf. Sci., 2015, 453, 186-193.

124. Singha, D., Sahu, D. K. and Sahu, K., Anomalous spectral modulation of 4-aminophthalimide inside acetonitrile/AOT/ $n$-heptane microemulsion: new Insights on reverse micelle to bicontinuous microemulsion transition. J. Phys. Chem. B, 2018, 122, 6966-6974.

125. Mukherjee, I., Dinda, G., Ghosh, S. and Moulik, S. P., Synthesis, characterization and applications of microheterogeneous templated CdS nano dispersions. J. Nanopart. Res., 2012, 14, 997-1011.

126. Capek, I., Preparation of metal nanoparticles in water-in-oil (w/o) microemulsions. Adv. Colloid Interf. Sci., 2004, 110, 49-74.

127. Mukherjee, I., Senapati, S., Mitra, D., Rakshit, A. K., Das, A. R. and Moulik, S. P., Physicochemistry of dispersions of $\mathrm{HgO}, \mathrm{HgS}$ and 'Makardhwaj' (an Ayurvedic medicine) prepared in micelle and microemulsion templates. Colloid Surface A, 2010, 360, 142149.

128. Gupta, S. and Moulik, S. P., Biocompatible microemulsions and their prospective uses in drug delivery. J. Pharm. Sci., 2008, 97, $22-45$.

129. Gupta, S., Moulik, S. P., Hazra, B., Ghosh, R., Sanyal, S. K. and Datta, S., New pharmaceutical microemulsion system for encapsulation and delivery of diospyrin, a plant-derived bioactive quinonoid compound. Drug Deliv., 2006, 13, 193-199.

130. Tenjarala, S., Microemulsions: an overview and pharmaceutical applications. Crit. Rev. Ther. Drug Carrier Syst., 1999, 16, 461521.

131. Boonme, P., Applications of microemulsions in cosmetics. $J$. Dermatol., 2007, 6, 223-228.

132. Azeem, A., Rizwan, M., Ahmad, F. J., Khan, Z. I., Khar, R. K., Aqil, M. and Talegaonkar, S., Emerging roles of microemulsions in cosmetics. Recent Pat. Drug Deliv. Formul., 2008, 2, 275-289.

133. Halloran, D. and Hoag, C., Organofunctional silicone microemulsions for hair formulations. J. Cosmet. Sci., 1998, 113, 61-68.

134. von Rybinski, W., Hloucha, M. and Johansson, I., Microemulsions (ed. Stubenrauch, C.), Wiley, Chichester, UK, 2009, chap. 8.

135. Hamdan, S., Dai, Y. Y. and Ahmed, F. B. H., Evaporation from microemulsion with perfume. Orient. J. Chem., 1997, 13, 111120.

136. Friberg, S. E., Vapour pressure of some fragrance ingredients in emulsion and microemulsion formulations. Int. J. Cosmet. Sci., 1997, 19, 75-86.

137. Kim, E. J., Kong, B. J., Kwon, S. S., Jang, H. N. and Park, S. N., Preparation and characterization of W/O microemulsion for removal of oily make-up cosmetics. Int. J. Cosmet. Sci., 2014, 36, 606-612.

138. Chellapa, P., Ariffin, F. D., Eid, A. M., Almahgoubi, A. A., Mohamed, A. T., Issa, Y. S. and Elmarzugi, N. A., Nanoemulsion for cosmetic applications. Eur. J. Biomed. Pharm. Sci., 2016, 3, $8-11$.

139. Chen, J., Ma, X., Yao, G., Zhang, W. and Zhao, Y., Microemulsion-based anthocyanin systems: effect of surfactants, cosurfactants, and its stability. Int. J. Food Prop., 2018, 21, 1152-1165.

140. Du, Z., Mao, X., Tai, X., Wang, G. and Liu, X., Preparation and properties of microemulsion detergent with linear medium chain fatty alcohols as oil phase. J. Mol. Liq., 2016, 223, 805-810. 


\section{REVIEW ARTICLE}

141. Tongcumpou, C. et al., Microemulsion formation and detergency with oily soils: III. Performance and mechanisms. J. Surfact. Deterg., 2005, 8, 147-156.

142. Nguyen, T. T. and Sabatini, D. A., Characterization and emulsification properties of rhamnolipid and sophorolipid biosurfactants and their applications. Int. J. Mol. Sci., 2011, 12, 1232-1244.

143. Ochoterena, R., Lif, A., Nyden, M., Andersson, S. and Denbratt, I., Optical studies of spray development and combustion of water-in-diesel emulsion and microemulsion fuels. Fuel, 2010, 89, 122-132.

144. Singh, P. J., Khurma, J. and Singh, A., Preparation, characterisation, engine performance and emission characteristics of coconut oil based hybrid fuels. Renew. Energy, 2010, 35, 2065-2070.
ACKNOWLEDGEMENTS. We thank Ms Ananya Rakshit and Dr Animesh Pan for assistance. We also thank Prof. D. O. Shah and Prof. Sunil Bhagwat in finding the photograph of Prof. Schulman. S.P.M. thanks Jadavpur University and Indian National Science Academy for infrastructural and other supports. B.N. acknowledges Centre for Surface Science (Physical Chemistry Section), Department of Chemistry, Jadavpur University for required facilities.

Received 12 October 2018; revised accepted 27 December 2018

doi: $10.18520 / \mathrm{cs} / \mathrm{v} 116 / \mathrm{i} 6 / 898-912$

\section{CURRENT SEIENGE \\ Display Advertisement Rates}

\begin{tabular}{|c|c|c|c|c|c|c|c|}
\hline India & \multicolumn{7}{|c|}{ Tariff (Rupees)* } \\
\hline \multirow[b]{2}{*}{ Size } & \multirow{2}{*}{$\begin{array}{c}\text { No. of } \\
\text { insertions }\end{array}$} & \multicolumn{2}{|c|}{ Inside pages } & \multicolumn{2}{|c|}{ Inside cover pages } & \multicolumn{2}{|c|}{ Back cover pages } \\
\hline & & B\&W & Colour & B\&W & Colour & B\&W & Colour \\
\hline \multirow{7}{*}{$\begin{array}{l}\text { Full page } \\
\left(\begin{array}{l}H=23 \mathrm{~cm} \\
W=17.5 \mathrm{~cm})\end{array}\right.\end{array}$} & 1 & 18,000 & 30,000 & 25,000 & 40,000 & 35,000 & 45,000 \\
\hline & 2 & 33,000 & 55,000 & 47,000 & 76,000 & 65,000 & 86,000 \\
\hline & 4 & 62,000 & $1,05,000$ & 92,000 & $1,46,000$ & $1,25,000$ & $1,65,000$ \\
\hline & 6 & 90,000 & $1,50,000$ & $1,25,000$ & $2,00,000$ & $1,75,000$ & $2,25,000$ \\
\hline & 8 & $1,10,000$ & $1,87,000$ & $1,68,000$ & $2,65,000$ & $2,30,000$ & $3,00,000$ \\
\hline & 10 & $1,35,000$ & $2,25,000$ & $1,98,000$ & $3,15,000$ & $2,70,000$ & $3,60,000$ \\
\hline & 12 & $1,80,000$ & $3,00,000$ & $2,50,000$ & $4,00,000$ & $3,50,000$ & $4,50,000$ \\
\hline \multirow{7}{*}{$\begin{array}{l}\text { Half page } \\
(\mathrm{H}=11 \mathrm{~cm} ; \\
W=17.5 \mathrm{~cm})\end{array}$} & 1 & 10,000 & 18,000 & \multirow{7}{*}{$\begin{array}{l}\text { Quarter page } \\
\begin{array}{l}(\mathrm{H}=11 \mathrm{~cm} ; \\
\text { W = 8 cm) }\end{array}\end{array}$} & \multirow{2}{*}{$\begin{array}{c}\text { No. of } \\
\text { insertions }\end{array}$} & \multicolumn{2}{|c|}{ Inside pages } \\
\hline & 2 & 19,000 & 33,000 & & & B\&W & Colour \\
\hline & 4 & 35,000 & 62,000 & & 1 & 6,000 & 12,000 \\
\hline & 6 & 50,000 & 90,000 & & 6 & 30,000 & 60,000 \\
\hline & 8 & 60,000 & $1,10,000$ & & 12 & 60,000 & $1,20,000$ \\
\hline & 10 & 72,000 & $1,35,000$ & & & & \\
\hline & 12 & $1,00,000$ & $1,80,000$ & & & & \\
\hline $\begin{array}{c}\text { Other } \\
\text { Countries }\end{array}$ & \multicolumn{7}{|c|}{ Tariff (US \$)* } \\
\hline \multirow[b]{2}{*}{ Size } & \multirow{2}{*}{$\begin{array}{c}\text { No. of } \\
\text { insertions }\end{array}$} & \multicolumn{2}{|c|}{ Inside pages } & \multicolumn{2}{|c|}{ Inside cover pages } & \multicolumn{2}{|c|}{ Back cover pages } \\
\hline & & B\&W & Colour & B\&W & Colour & B\&W & Colour \\
\hline \multirow{2}{*}{$\begin{array}{l}\text { Full page } \\
(\mathrm{H}=23 \mathrm{~cm} ; \\
\mathrm{W}=17.5 \mathrm{~cm})\end{array}$} & 1 & 300 & 650 & 450 & 750 & 600 & 1000 \\
\hline & 6 & 1500 & 3000 & 2250 & 3500 & 3000 & 5000 \\
\hline \multirow{2}{*}{$\begin{array}{l}\text { Half page } \\
(H=11 \mathrm{~cm} ; \\
W=17.5 \mathrm{~cm})\end{array}$} & 1 & 200 & 325 & & & & \\
\hline & 6 & 1000 & 2000 & & & & \\
\hline
\end{tabular}

${ }^{*} 25 \%$ rebate for Institutional members

Note: For payments towards the advertisement charges, Cheque (at par/multicity) or Demand Drafts may be drawn in favour of 'Current Science Association, Bengaluru'.

Contact us: Current Science Association, C.V. Raman Avenue, P.B. No. 8001, Bengaluru 560080 or e-mail: csc@ias.ac.in

Last date for receiving advertising material: Ten days before the scheduled date of publication.

[The jurisdiction for all disputes concerning submitted articles, published material, advertisement, subscription and sale will be at courts/tribunals situated in Bengaluru city only.] 